\title{
Kahook Dual Blade Goniotomy vs iStent inject: Long-Term Results in Patients with Open-Angle Glaucoma
}

This article was published in the following Dove Press journal: Clinical Ophthalmology

\author{
Thorsteinn S Arnljots (D)' \\ Mario A Economou ${ }^{2,3}$ \\ 'Anterior Segment Department, St. Erik \\ Eye Hospital, Stockholm, Sweden; \\ ${ }^{2}$ Division of Ophthalmology and Vision, \\ Department of Clinical Neuroscience, \\ Karolinska Institutet, Stockholm, Sweden; \\ ${ }^{3}$ Department of Ophthalmology, \\ Sophiahemmet Hospital, Stockholm, \\ Sweden
}

Purpose: Efficacy and safety evaluation of Kahook Dual Blade (KDB) goniotomy vs iStent inject implantation.

Materials and Methods: Retrospective study in patients that underwent goniotomy with KDB or iStent inject implantation, stand-alone or combined with cataract surgery. Main outcome parameters were intraocular pressure (IOP), number of glaucoma medications, proportion of eyes achieving $>20 \%$ IOP reduction and number of eyes with postoperative IOP $<19 \mathrm{mmHg}$ at last follow-up.

Results: A total of 29 patients (30 eyes) were included in the iStent inject group and 30 patients (32 eyes) in the KDB group. Mean follow-ups were 20.9 \pm 6.5 (KDB-alone) to 29.5 \pm 7.6 (phaco-iStent inject) months. Pre- and post-operative IOPs were $22.2 \pm 5.8 \mathrm{mmHg}$ and $15.9 \pm 4.3 \mathrm{mmHg}(P=0.004)$ in the KDB-alone, $24.2 \pm 6.8 \mathrm{mmHg}$ and $16.2 \pm 6.7 \mathrm{mmHg}$ $(P=0.001)$ in the phaco-KDB, $20.6 \pm 5.4 \mathrm{mmHg}$ and $20.9 \pm 6.8 \mathrm{mmHg}(P=0.598)$ in the iStent inject-alone as well as $20.9 \pm 5.5 \mathrm{mmHg}$ and $15.6 \pm 3.4 \mathrm{mmHg}(P=0.003)$ in the phacoiStent inject subgroups. No major complications occurred.

Conclusion: All KDB and iStent subgroups except the stand-alone iStent inject subgroup showed a clinically significant IOP-lowering effect as a stand-alone procedure or combined with cataract surgery. Goniotomy with KDB in this setting seems to offer an advantageous IOP reduction compared to iStent inject.

Keywords: MIGS, iStent inject, Kahook Dual Blade, glaucoma surgery, goniotomy

\section{Introduction}

Glaucoma is the world's leading cause of irreversible blindness and the number of people affected is expected to increase dramatically over the next decades. ${ }^{1}$ Consequently, demand for effective treatment options will become increasingly important in the future. In recent years increasing attention has been focused into the development of micro-invasive glaucoma surgery (MIGS) with the aim to provide a clinically significant reduction of intraocular pressure (IOP) along with an improved safety profile. These techniques broadly target the aqueous outflow through the Schlemm's canal, the subconjunctival or the suprachoroidal space. ${ }^{2}$

The iStent (Glaukos Corp, Laguna Hills, CA, USA) micro-implant received its CE mark in 2004 and FDA approval in 2012. It is inserted through an ab interno approach into the trabecular meshwork (TM) thereby improving aqueous outflow through the Schlemm's canal. ${ }^{3}$ The iStent inject is the second generation of trabecular micro-bypass stents and utilizes an auto-injector mechanism to implant
Correspondence: Thorsteinn S Arnljots Sankt Eriks Ögonsjukhus, Eugeniavägen

12, Solna, Stockholm, 17| 64

Tel +46-8-672 3063

Email thorsteinn.snaebjornsson-

arnljots@sll.se 
two devices instead of only one through the TM into the Schlemm's canal. ${ }^{2}$ The iStent inject has shown efficacy both when performed as a stand-alone procedure as well as in combination with phacoemulsification cataract surgery. ${ }^{4,5}$

The single-use goniotomy Kahook Dual Blade (KDB, New World Medical Inc, Rancho Cucamonga, CA, USA) is a more recent surgical treatment modality that also targets the trabecular outflow but with a slightly different approach. The device received its FDA approval in 2015 and has also shown good efficacy, both as a stand-alone procedure or combined with cataract surgery. ${ }^{6-8}$ The device is introduced into the anterior chamber (AC) through a temporal clear corneal incision and after piercing TM with the tip of the device it is advanced forward along the length of the TM. The inbuilt ramp of the device tip elevates trabecular tissue which is then excised by the dual parallel blades. Contrary to the Trabectome (Neomedix Corp, Tustin, CA, USA), which makes an incision into and causing evaporation in the $\mathrm{TM},{ }^{9}$ the KDB technique also involves removing the excised TM which in theory reduces the risk of scarring and subsequent failure. ${ }^{8}$

To the authors' knowledge, although studies comparing KDB with the first-generation iStent already exist, this is the first head-to-head comparison including the second generation of iStent known as iStent inject. The purpose of this study was to evaluate and compare the efficacy as well as safety profile of KDB goniotomy and iStent inject when performed either as an isolated procedure or in combination with phacoemulsification cataract surgery.

\section{Materials and Methods Study Design and Population}

This study was a retrospective, non-randomized, noncontrolled, interventional case series. Data were collected from medical records of eyes with open-angle glaucoma that underwent elective goniotomy with $\mathrm{KDB}$ or iStent inject implantation either as a stand-alone procedure (KDB/iStent inject-alone) or in combination with phacoemulsification cataract surgery (phaco-KDB/iStent inject). All patients that underwent these procedures between October 2016 and February 2019 at St. Erik Eye Hospital in Stockholm, Sweden, were included in the analysis and were operated on by a single experienced surgeon (M.A.E.) who had initially performed at least 40 cases of iStent inject and KDB during his training period that were not included in the study.
Preoperative assessment and data collection included a complete review of the patient's ophthalmic history, Goldmann applanation IOP (without medication washout), Snellen best-corrected visual acuity (BCVA) at 20 feet under standardized lighting conditions (converted to $\log$ MAR visual acuity in analysis for ease of interpretation), indirect gonioscopy, non-dilated and dilated slit lamp biomicroscopy examination of the anterior and posterior segment (90D lens) respectively, as well as standard automatic perimetry using the Humphrey ${ }^{\circledR}$ Field Analyzer 3 (Carl Zeiss Meditec Inc, Dublin, CA, USA).

Inclusion criteria were mild to moderate (according to current ICD-10 classification) open-angle glaucoma (as confirmed by gonioscopy) or ocular hypertension, pseudophakic or phakic with a clear lens (in the case of standalone procedures) or as a combined surgery, preoperative IOP (without washout) between 15 and $35 \mathrm{mmHg}$ as well as age $>18$ years. Exclusion criteria were patients with corneal opacities not permitting adequate viewing conditions of the angle and clinically significant peripheral synechiae/closed-angle glaucoma.

Indications for both surgeries were similar most commonly including suboptimal IOP and/or slow visual field progression in spite of maximal tolerable glaucoma therapy with or without previous treatment with selective laser trabeculoplasty. Another relevant surgery group included patients on two to three types of topical glaucoma drops without known drop intolerance requiring further IOP reduction that were otherwise scheduled for cataract surgery and wished to further reduce the drop load. The choice between iStent inject or KDB goniotomy was based on the surgeon's preferences and a detailed discussion with the patient whereby an informed consent was obtained in all of the cases.

The protocol of this study was approved by the Institutional Review Board (IRB) of the Swedish Ethical Review Authority (2019-03468) and adhered to the ethical tenets of the Declaration of Helsinki. The study being retrospective in nature and all information de-identified informed consent was not required by the IRB.

\section{Surgical Technique}

Surgical technique was applied as previously described in the case of $\mathrm{KDB}$ goniotomy as well as iStent inject implantation. ${ }^{6,10}$ In all surgeries combined with cataract surgery, phacoemulsification and intraocular lens implantation was performed first in a standardized routine fashion. After cataract surgery, $1 \%$ acetylcholine was 
administered into the $\mathrm{AC}$ to constrict the pupil. Subsequently, microstent implantation or KDB goniotomy was performed as described below.

\section{Goniotomy with Kahook Dual Blade}

Briefly, the AC was filled with cohesive viscoelastic then the $\mathrm{KDB}$ was advanced into the $\mathrm{AC}$ through a $1.8 \mathrm{~mm}$ temporal clear corneal incision. Under direct gonioview the distal tip was allowed to pierce the TM and enter the Schlemm's canal. By virtue of its construction, the footplate ramp directly elevates the TM when being advanced along the desired length of the treatment area which allows the parallel dual blades to excise tissue in a standardized fashion. Finally, the freely moving TM tissue was removed using a pair of vitreoretinal micro-forceps. All remaining viscoelastic was then removed with irrigation/aspiration. Usually, a smaller amount of blood reflux emanating from the collector channels was observed, indicating a patent aqueous outflow.

\section{iStent inject}

In the stand-alone procedure, the $\mathrm{AC}$ was entered through a temporal $1.8 \mathrm{~mm}$ clear corneal incision. The AC was prefilled with viscoelastic followed by implantation of two devices two to three clock hours apart in the nasal quadrant.

\section{Follow-Up}

Follow-up was routinely scheduled with typically the first postoperative visit on day one, then two weeks, one month, three months, six months, nine months, one year, 15 months, 18 months and two years depending on clinical status. All follow-up visits included assessment of number and type of glaucoma medications, standard BCVA, Goldmann IOP (without washout), indirect gonioscopy as well as anterior and posterior segment (90D) slit lamp examination. Visual field examinations were performed depending on the clinical needs.

Typically, patients in the stand-alone procedures were given a postoperative topical therapy of $0.3 \%$ nepafenac once daily for three to four weeks except in risk groups for postoperative cystoid macular edema in which case a six weeks' course was administered. However, in the combined surgeries topical preservative-free $0.1 \%$ dexamethasone was typically prescribed instead, three times daily then tapered during a standard three weeks' course. The surgeon continued administration of IOP-lowering medications after the surgery, and the first decision to discontinue some of them was taken six to eight weeks after surgery.

\section{Outcome Measures}

Primary outcome measures were pre- and postoperative IOPs, number of patients with $>20 \%$ postoperative IOP reduction and number of patients with postoperative IOP $<19 \mathrm{mmHg}$ at last follow-up. These cut-offs were chosen to reflect and match previous studies focusing on the same surgical techniques. Secondary outcome measures were pre- and postoperative number of glaucoma medications and BCVA at last follow-up as well as complications related to the procedures.

\section{Statistical Analysis}

Statistical analysis was performed with GraphPad Prism 8.4.1 (GraphPad Software Inc, La Jolla, CA, USA). Normality of data distribution was tested with D'Agostino-Pearson's normality test. In the case of normally distributed data a paired $t$ test was used and in the case of non-normally distributed data the Wilcoxon matched-pairs signed-rank test was used. When analyzing non-paired data un-paired $t$ test was used in the case of normally distributed data and Mann-Whitney $U$-test in the case of not normally distributed data. The Kruskal-Wallis test was applied to detect significant baseline differences in age, BCVA, visual field results, IOP and number of glaucoma medications. Results were presented as mean \pm standard deviation (SD). Statistical significance level was set at $P<0.05$.

\section{Results}

The clinical and demographical patient characteristics are provided in Table 1. A total of 62 eyes (59 patients) were included in the study. Twenty-nine patients (30 eyes) underwent stand-alone or combined iStent inject implantation and 30 patients (32 eyes) underwent goniotomy with KDB. Mean follow-ups in the iStent inject-alone, combined iStent inject, KDB-alone and combined KDB subgroups were $22.0 \pm 10.8$ (median 23.7, range 2.6-37.6), $29.5 \pm 7.6$ (median 30.9, range 10.7-40.2), 20.9 \pm 6.5 (median 22.7, range 11.4-30.5) and 21.8 \pm 7.1 (median 22.3, range 12.0-34.3) months, respectively. All patients reached a minimum of $\geq 12$ months of follow-up except three eyes in the stand-alone iStent inject subgroup that failed within the first year (defined as failures based on subsequently undergoing secondary ocular surgery, see below). One patient in the iStent inject-alone subgroup 
Table I Preoperative Baseline Demographics and Clinical Characteristics in Patients Undergoing Kahook Dual Blade (KDB) Goniotomy or iStent inject Implantation as Sole Procedures (-Alone) or in Combination with Standard Phacoemulsification Cataract Surgery (Phaco-)

\begin{tabular}{|c|c|c|c|c|c|c|}
\hline Parameters & & KDB-Alone & Phaco-KDB & iStent inject-Alone & Phaco-iStent inject & $P$ value \\
\hline No. of eyes & & 13 & 19 & 14 & 16 & \\
\hline \multicolumn{2}{|c|}{ Mean age (years $\pm S D$ ) } & $62.0 \pm 13.9$ & $66.5 \pm 9.0$ & $72.0 \pm 12.1$ & $77.7 \pm 6.7$ & 0.138 \\
\hline \multicolumn{7}{|l|}{ Gender } \\
\hline & Female & 7 & 11 & 13 & 10 & \\
\hline & Male & 6 & 8 & I & 6 & \\
\hline \multicolumn{7}{|c|}{ Glaucoma diagnosis } \\
\hline & POAG & 2 & 9 & 6 & 3 & \\
\hline & PXG & 8 & 7 & 5 & 12 & \\
\hline & PDG & 2 & 1 & & & \\
\hline & NTG & & & & I & \\
\hline & OHT & I & 2 & 3 & & \\
\hline \multicolumn{7}{|c|}{ Preoperative lens status } \\
\hline & Phakic & 5 & & 5 & & \\
\hline & Pseudophakic & 8 & & 9 & & \\
\hline \multicolumn{2}{|c|}{ Mean BCVA $\pm S D$, logMAR (median) } & $0.02 \pm 0.10(0.00)$ & $0.14 \pm 0.19(0.10)$ & $0.13 \pm 0.15(0.10)$ & $0.20 \pm 0.19(0.20)$ & 0.034 \\
\hline \multicolumn{2}{|c|}{ Mean visual field $\pm S D, M D$ (median) } & $-5.98 \pm 3.09(-5.31)$ & $-6.30 \pm 5.44(-2.75)$ & $-9.34 \pm 4.20(-9.83)$ & $-8.27 \pm 4.67(-7.22)$ & 0.309 \\
\hline \multicolumn{2}{|c|}{ Mean IOP \pm SD, mmHg } & $22.2 \pm 5.8$ & $24.2 \pm 6.8$ & $20.6 \pm 5.4$ & $20.9 \pm 5.5$ & 0.265 \\
\hline \multicolumn{2}{|c|}{ Mean no. of glaucoma medications $\pm S D$} & $3.2 \pm 1.6$ & $2.9 \pm 1.6$ & $3.0 \pm 1.1$ & $2.9 \pm 1.1$ & 0.905 \\
\hline
\end{tabular}

Abbreviations: BCVA, best-corrected visual acuity; IOP, intraocular pressure; NTG, normal-tension glaucoma; OHT, ocular hypertension; PDG, pigment dispersion glaucoma; POAG, primary open-angle glaucoma; PXG, pseudoexfoliative glaucoma; SD, standard deviation.

and two patients in the KDB-alone subgroup had undergone previous glaucoma surgery.

\section{Intraocular Pressure Outcomes KDB Goniotomy}

Pre- and postoperative IOPs at last follow-up in the single procedure subgroup were $22.2 \pm 5.8 \mathrm{mmHg}$ and $15.9 \pm 4.3$ $\mathrm{mmHg}$, resulting in a mean IOP reduction of $6.3 \pm 6.4$ $\mathrm{mmHg}$ or $28.4 \%(P=0.004)$. Pre- and postoperative IOPs in the combined subgroup were $24.2 \pm 6.8 \mathrm{mmHg}$ and 16.2 $\pm 6.7 \mathrm{mmHg}$, resulting in a mean IOP reduction of $7.9 \pm 8.2$ $\mathrm{mmHg}$ or $32.0 \%(P=0.001)$. Success rates at the $<19$ $\mathrm{mmHg}$ and $>20 \%$ IOP reduction levels were $77 \%$ and $62 \%$ in the KDB-alone and $79 \%$ and $84 \%$ in the combined subgroups, respectively. Two patients had undergone previous glaucoma surgery (deep sclerectomy and trabeculectomy) and showed an IOP reduction at last follow-up of $24-50 \%$ with a reduction of one medication class.

\section{iStent inject}

Baseline and postoperative IOPs at last follow-up in the standalone subgroup were $20.6 \pm 5.4 \mathrm{mmHg}$ and $20.9 \pm 6.8 \mathrm{mmHg}$, rendering a mean increase of $0.3 \pm 6.5 \mathrm{mmHg}$ or $1.5 \%$ $(P=0.598)$. Excluding five outliers resulted in mean pre- and postoperative IOPs of $18.7 \pm 1.6$ and $16.2 \pm 2.6 \mathrm{mmHg}$ as well as a mean decrease of $2.4 \pm 3.0 \mathrm{mmHg}$ or $13.1 \%(P=0.043)$. Apart from this subanalysis, all other statistical analysis and graphs include all patients as well as outliers. Baseline and postoperative IOPs in the combined subgroup were $20.9 \pm 5.5$ $\mathrm{mmHg}$ and $15.6 \pm 3.4 \mathrm{mmHg}$, rendering a mean reduction of $5.3 \pm 6.1 \mathrm{mmHg}$ or $25.4 \%(P=0.003)$. One patient had undergone previous glaucoma surgery (deep sclerectomy) and showed an IOP reduction of $30 \%$ at last follow-up with no change in number of glaucoma medications. Primary endpoint success rates at the $<19 \mathrm{mmHg}$ and $>20 \%$ IOP reduction levels were $57 \%$ and $29 \%$ in the stand-alone procedure and $71 \%$ and $59 \%$ in the combined subgroups, respectively.

In a between all subgroups-analysis, significant differences in IOP reduction $(\mathrm{mmHg})$ at last follow-up were observed between the stand-alone iStent inject vs phacoiStent inject $(P=0.015)$, iStent inject-alone vs KDB-alone $(P=0.046)$ as well as the iStent inject-alone vs phaco-KDB $(P=0.000)$ subgroups. Figure 1 summarizes the changes in 


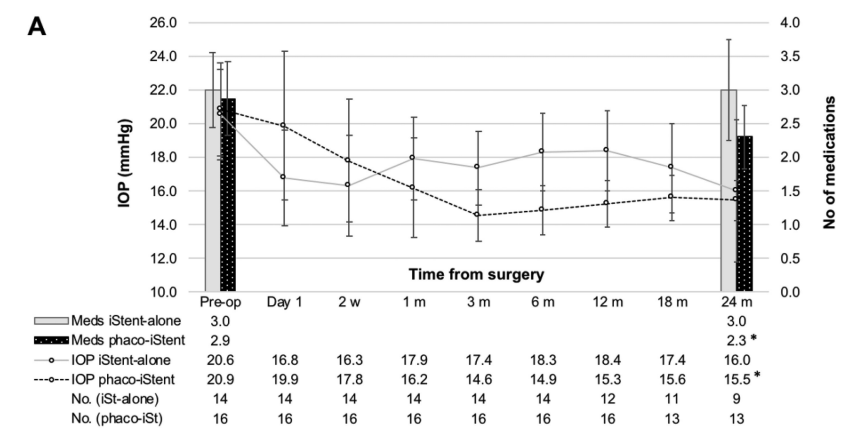

C

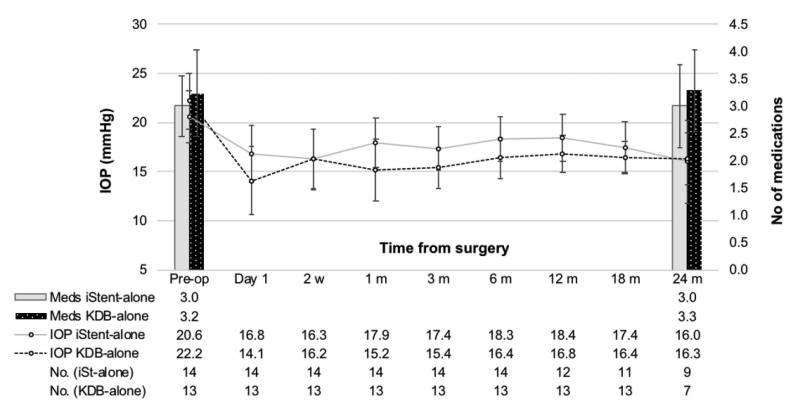

B

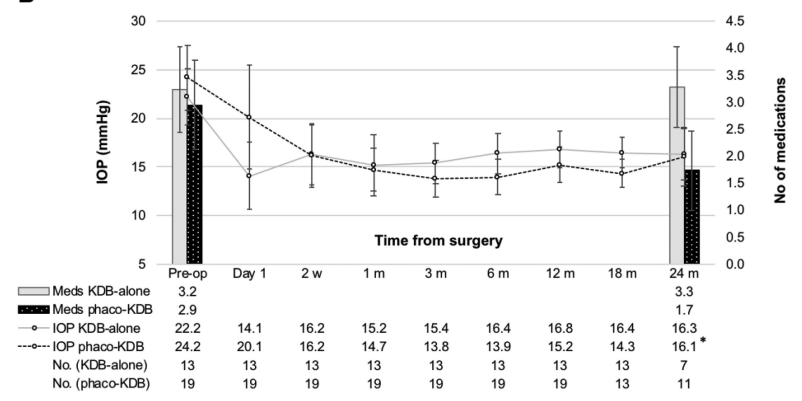

D

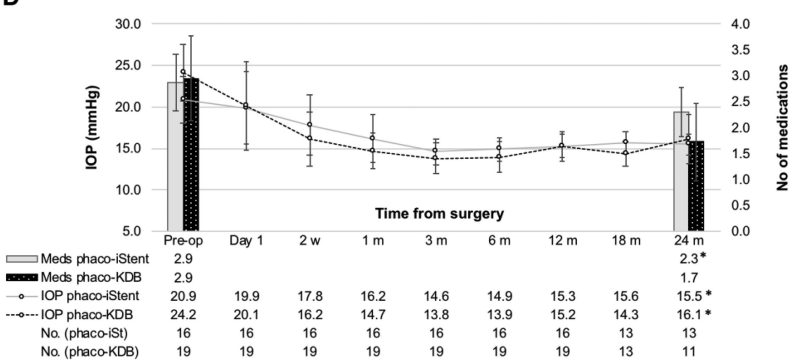

Figure I Intraocular pressure and number of IOP-lowering medications throughout the first 24 months of follow-up with KDB goniotomy and iStent inject implantations, categorized as stand-alone procedures or combined with phacoemulsification cataract surgery (error bars representing SD). A statistically significant IOP-lowering at 24 months compared to baseline was observed in the phaco-KDB and phaco-iStent inject subgroups $(P=0.002$ and $P=0.002$, respectively), non-significantly so in the KDB-alone and iStent injectalone subgroups $(P=0.094$ and $P=0.109$, respectively). The phaco-iStent inject subgroup demonstrated a statistically significant lowering of number of glaucoma medications at 24 months $(P=0.01 \mathrm{I})$ while the KDB-alone, phaco-KDB and iStent inject-alone subgroups did not $(P=0.750, P=0.104$ and $P=0.45 \mathrm{I}$, respectively). (A) iStent inject-alone vs phaco-iStent inject implantation; (B) Kahook Dual Blade goniotomy single procedure vs phaco-KDB; (C) iStent inject-alone vs KDB-alone; (D) Phaco-iStent inject vs phaco-KDB.

Note: $* P<0.05$.

Abbreviations: IOP, intraocular pressure; KDB, Kahook Dual Blade; Meds, number of medications; SD, standard deviation.

IOP and number of glaucoma medication over the course of the initial 24 months of follow-up comparing the different subgroups. Figure 2 shows a direct comparison of IOP reduction between all subgroups at the 24-month follow-up as well as last follow-up. Figure 3 shows the primary outcome measure success rates (\%) at last followup, comparing all study subgroups.

\section{Number of Glaucoma Medications KDB Goniotomy}

Number of medications in the stand-alone subgroup at baseline and last follow-up were $3.2 \pm 1.6$ and $2.8 \pm 1.2$, resulting in a mean reduction of $0.5 \pm 1.5$ medications or $14.3 \%(P=0.274)$. In the combined surgery subgroup, the number of medications at baseline and last follow-up were $2.9 \pm 1.6$ and $2.3 \pm 1.5$, respectively, a mean reduction of 0.6 \pm 1.1 or $21.5 \%(P=0.024)$.

\section{iStent inject}

Pre- and postoperative number of medications in the standalone subgroup increased from $3.0 \pm 1.1$ to $3.3 \pm 1.4$ $(P=0.165)$. Pre- and postoperative number of medications in the combined subgroup were $2.9 \pm 1.1$ and $2.1 \pm 0.9$, representing a mean reduction of $0.8 \pm 1.1$ or $28.2 \%(P=0.010)$.

\section{Safety}

Mean pre- and postoperative BCVA remained statistically unchanged in all subgroups from a baseline of $0.13 \pm 0.15$ $\log$ MAR to $0.15 \pm 0.121 \log$ MAR $(P=0.906), 0.20 \pm 0.19$ $\log$ MAR to $0.10 \pm 0.26 \log$ MAR $(P=0.051), 0.02 \pm 0.10$ $\log$ MAR to $0.08 \pm 0.14 \log$ MAR $(P=0.131)$ and $0.14 \pm 0.19$ $\log$ MAR to $0.05 \pm 0.10 \log$ MAR $(P=0.058)$ in the iStentalone, phaco-iStent, KDB-alone as well as the phaco-KDB subgroups, respectively. Thus, none of the subgroups showed a statistically significant decrease in BCVA nor were any cases of loss of light perception observed.

In the KDB-alone subgroup, one eye experienced cystoid macular oedema (CMO) one month postoperatively that showed a complete regression following a six weeks' course of $0.1 \%$ nepafenac. ${ }^{11}$ Another eye experienced intraoperative iris prolapse resulting in moderate temporal iris atrophy.

In the combined KDB subgroup, one eye experienced an IOP-spike on postoperative day one (45 $\mathrm{mmHg}$ ) due to retained viscoelastic, relieved with an 

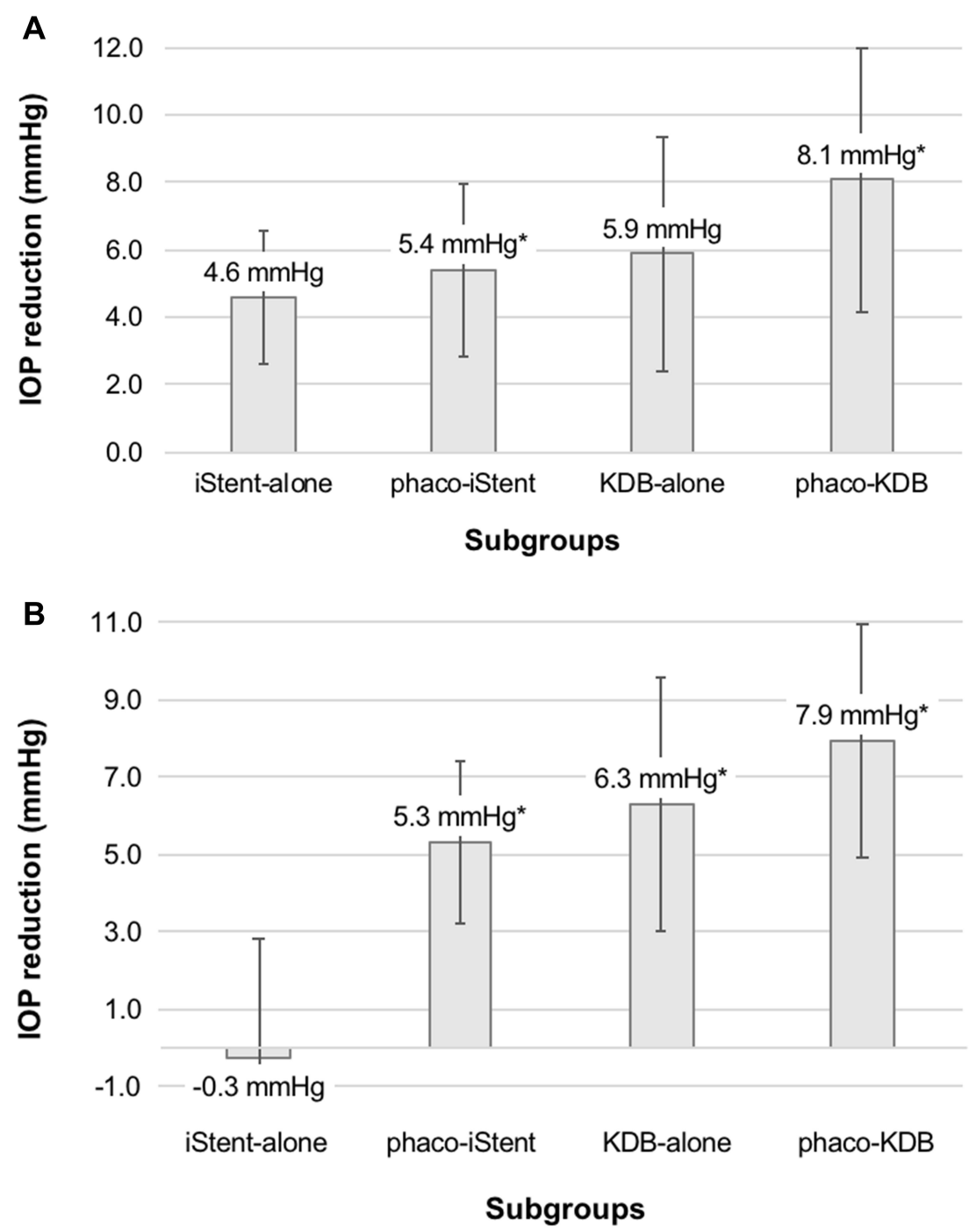

Figure 2 Subgroup comparison of IOP reduction $(\mathrm{mmHg})$ from baseline showing the differences between iStent inject-alone, phaco-iStent inject, KDB goniotomy-alone and phaco-KDB. (A) A statistically significant mean IOP reduction (error bars indicating $\pm S D$ ) at 24 months compared to baseline was observed in the combined KDB and iStent inject subgroups $(P=0.002$ and $P=0.002$, respectively); $(B)$ At last follow-up, the KDB-alone $(P=0.004)$, phaco-KDB $(P=0.00 I)$ and phaco-iStent inject $(P=0.003)$ subgroups all showed a statistically significant mean reduction of IOP while the iStent inject-alone group $(P=0.598)$ failed to do so. Mean follow-up $\pm S D$ in brackets.

Note: $* P<0.05$

Abbreviations: IOP, intraocular pressure; KDB, Kahook Dual Blade; SD, standard deviation.

AC tap. Two cases of transient postoperative corneal edema occurred, treated with $5 \%$ sodium chloride ointment. One of these eyes also demonstrated temporary postoperative hyphema as well as short-lasting postoperative hypotony $<5 \mathrm{mmHg}$ without choroidal bullae nor further sequelae.

In the iStent inject-alone subgroup, one case of severe $\mathrm{CMO}$ occurred in an eye with a previously known diagnosis of central retinal vein occlusion due to which the patient had previously undergone panretinal photocoagulation as well as intravitreal injections of aflibercept. Also, one case of intraoperative iris prolapse occurred.
In the combined iStent inject subgroup there was one case of mild, transient postoperative corneal oedema. One eye demonstrated a postoperative IOP spike due to retained viscoelastic device. Another eye was initially scheduled for KDB goniotomy however due to excessive patient mobility intraoperatively it was decided to implant two iStent inject instead.

Blood reflux emanating from the collector channels is a common occurrence in angle surgery, as was noted most commonly in the goniotomy subgroups but also in a majority of the microstent subgroups. Usually, only a small amount of hyphema could be detected in the inferior AC angle on the 


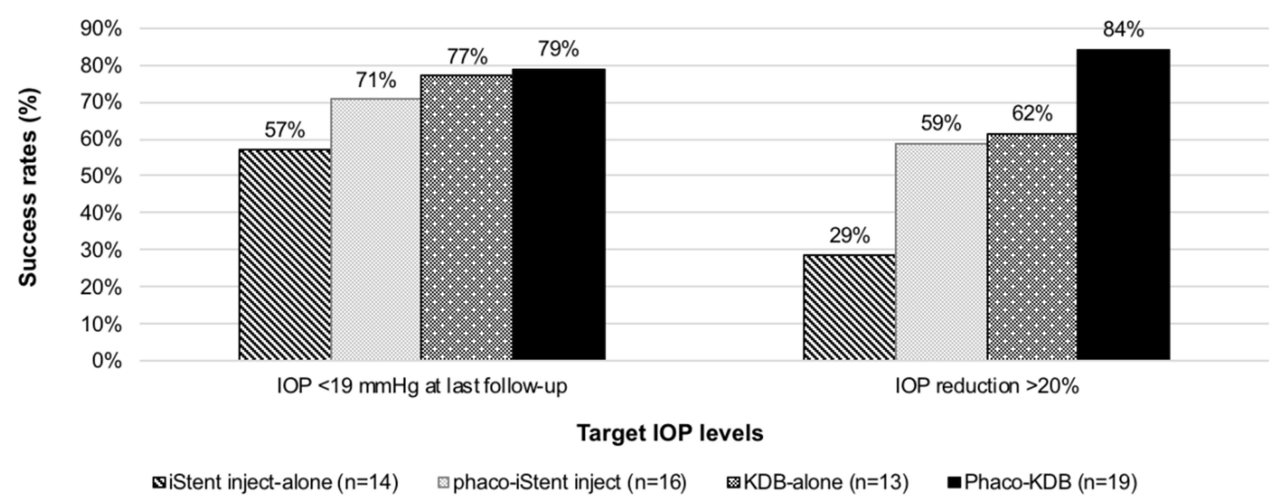

Figure 3 Success rates (\% of all patients per subgroup) at the target IOP level of $<19 \mathrm{mmHg}$ as well as proportional IOP reduction of $>20 \%$ from baseline to last follow-up, comparing all study subgroups (KDB goniotomy as a single procedure, combined phaco-KDB, iStent inject as a stand-alone procedure and phaco-iStent inject). Abbreviations: IOP, intraocular pressure; KDB, Kahook Dual Blade.

postoperative day one visit. The residual blood was completely resolved in all eyes at the one month-visit.

Secondary ocular surgery was performed in six eyes in the iStent inject-alone subgroup due to inadequate intraocular pressure control whereof three eyes year 0-1 (two trabeculectomies, one KDB), two eyes year 1-2 (one trabeculectomy, one PRESERFLO ${ }^{\mathrm{TM}}$ MicroShunt) and one eye year 2-3 (trabeculectomy). One eye in the phaco-KDB required subsequent filtering surgery (year 1-2), none in the other subgroups.

\section{Discussion}

Aiming to narrow the historical gap between non-surgical (medications and laser treatment) and traditional surgical glaucoma therapy in the glaucoma treatment paradigm, the field of MIGS is continuously expanding and newer techniques have been explored. ${ }^{2}$ However, although a certain number of studies already exist and continue to appear on different individual techniques there is still a great clinical need of comparisons between different procedures. This study presents relevant clinical data directly comparing two important MIGS technologies, KDB goniotomy and iStent inject, which to the authors' knowledge has not been reported until now.

In this study, the combined phaco-KDB subgroup showed a tendency towards favorable IOP-lowering results compared to not only the combined iStent inject subgroup but also the stand-alone subgroups, not only at last followup and 24 months but also as a proportion reaching $>20 \%$ IOP reduction. Furthermore, the single KDB subgroup seemed to outperform both the iStent inject subgroups. However, it must be borne in mind that preoperative IOP was higher in both the KDB groups, in turn possibly allowing a larger dynamic change in IOP.

The positive findings in the single KDB subgroup draw specific attention considering that a large proportion of these eyes had pseudoexfoliative glaucoma, which is often considered to impact prognosis negatively. Previous studies on excisional goniotomy with KDB have reported IOP reductions of $-31 \%$ as a stand-alone procedure and from $-14 \%$ to $-28 \%$ in combined surgeries. ${ }^{12-16}$ In the same studies, reported reductions in medication burden were $-26 \%$ in stand-alone surgery and $-21 \%$ to $-70 \%$ in the combined subgroups. In this study, IOP reductions of $-28.4 \%$ in the stand-alone and $-32.0 \%$ in the combined subgroups were observed along with a medication reduction of $-14.3 \%$ and $-21.5 \%$, respectively. Considering the significantly longer follow-up in this study, it comes as no surprise that the reduction in medication burden locates in the lower interval in comparison. On the other hand, IOP reductions were mainly consistent with previous reports, indicating a lasting IOP-lowering effect throughout not only the first but also the second year of follow-up.

Regarding the iStent inject, current literature showed an IOP-lowering in the range of $-31 \%$ to $-48 \%$ (with washout) as a sole procedure and $-28 \%$ to $-37 \%$ when combined with cataract surgery. ${ }^{4,5,17-21}$ The current study showed a pressure-lowering effect slightly lower than in previous reports in the combined subgroup. This might be due to different study populations, absence of washout IOPs (which possibly increases the IOP-lowering potential), the relatively small sample size in this study as well as the significantly longer follow-up in this study. Interestingly, in the present study, the stand-alone iStent inject subgroup had a predominant proportion of surgical 
failures (six of 14 eyes in the subgroup, 43\%). Since the last IOP reading directly preceding the secondary surgery in these cases were usually $\sim 30 \mathrm{mmHg}$ and since the last observations were not being carried forward in the statistical analysis, this significantly and mainly impacted the preoperative vs last follow-up analysis. On the other hand, it might be noted that when not failing at two years, the remaining eyes actually did result in an IOP reduction of $22.3 \%$. The iStent inject-alone subgroup constituted more severe glaucoma (MD $-9.34 \pm 4.20$ vs $-5.98 \pm 3.09$ to -8.27 $\pm 4.67, P=0.309$ ). Also, the failing eyes had a higher number of preoperative IOP-lowering medications compared to the non-failing eyes ( $3.7 \pm 0.8$ vs $2.5 \pm 1.1, P=0.085$ ), possibly contributing to risk for failure. Thus, in a larger study, these failures might not have had the same impact considering the relatively low number of study participants in this study. The only remaining failure occurred in the phaco-KDB subgroup, however, with a preoperative IOP $26 \mathrm{mmHg}$ on five medication classes.

Whether the results in this study can be sustained over an even longer follow-up period will be a matter of great interest. Although data is slowly emerging studies on KDB goniotomy with longer follow-up are scarce, while Arriola-Villalobos et al already demonstrated an excellent IOP reduction of $36.9 \%$ at a long mean follow-up of 47.4 months after phaco-iStent inject. ${ }^{5}$

A recent 12-month retrospective comparison between combined KDB and the first generation iStent showed a superior mean IOP reduction (standard error) in the phaco-KDB group of $-5.0(0.3) \mathrm{mmHg}$ vs $-2.3(0.4)$ $\mathrm{mmHg}(P<0.001)$ in the combined iStent group but with a similar reduction in mean medication. ${ }^{14}$ Both procedures were performed in eyes with mild to moderate glaucoma, with comparable safety profiles. In the present study, larger IOP reductions were observed at a significantly longer follow-up while also favoring the combined excisional goniotomy $(-7.9 \pm 8.2 \mathrm{mmHg}, P=0.001)$ over the iStent inject $(-5.3 \pm 6.1 \mathrm{mmHg}, P=0.003)$ group. However, the referenced study had significantly lower preoperative IOPs in both groups as well as shorter follow-up. Also, the results are not fully comparable since the secondgeneration iStent with two devices implanted has already been shown to offer a larger IOP reduction compared to the first generation iStent. ${ }^{4}$ On a similar note, previous studies have also shown that multi-stent implantations may result in incremental IOP reduction compared to single-stent implantation. ${ }^{22}$
A study by Le et al compared phaco-iStent and phacoKDB with at least 12 months of follow-up. ${ }^{15}$ They found a mean IOP reduction in the iStent group from $17.6 \pm 4.8$ to $15.9 \pm 2.9 \mathrm{mmHg}(P=0.01)$ and $17.4 \pm 4.3$ to $15.0 \pm 4.0 \mathrm{mmHg}$ $(P=0.01)$ in the KDB group. Both resulted in a reduction in IOP and number of medications at 1 year. A large retrospective study comparing combined iStent (198 eyes) and KDB (237 eyes) found a significantly greater mean IOP reduction in the KDB group of $-4.2 \mathrm{mmHg}(23.7 \%)$ vs iStent $-2.7 \mathrm{mmHg}(16.4 \%)(P=0.001)$ along with a greater mean reduction in medications of 1.1 vs 0.9 , respectively $(P=0.001) .{ }^{16}$ Also, the phaco-KDB group had a lower number of IOP spikes of $6.3 \%$ vs $12.6 \%$ in the phaco-iStent group. Again, preoperative IOPs were significantly lower in the study with a more limited follow-up. Iwasaki et al directly compared combined KDB and iStent and found that the overall IOP percentage reduction was higher in the KDB group (26\%) vs the iStent group $(19 \%){ }^{23}$ The KDB group also had a higher success rate at 12 months after surgery while the iStent group showed a better safety profile with only a $2.3 \%$ occurrence rate of hyphema vs $16.3 \%$ in the KDB group. Thus, in most studies excisional goniotomy with the KDB seems to show an advantage in IOP-lowering capacity, as well as permitting a larger reduction in glaucoma medication burden.

In the safety analysis, only minor complications were observed. One case of hypotony occurred in the phaco-KDB subgroup, however transient and without sequelae. As has been consistently shown in previous studies, the most common complication after KDB goniotomy as well as the iStent inject technique is intra- and postoperative hyphema. ${ }^{6,7,12,16,18,20}$ The bleedings in this study were of minor degree, did not interfere with the surgeon's intraoperative viewing conditions, did not cause any increase in IOP and did not persist >one month. However, blood emanating from the collector channels is generally also regarded as one of the important clinical signs of a successful procedure. Regarding BCVA, one could possibly have expected a significant improvement in the combined subgroups. Even if a tendency towards improvement was seen this was not statistically significant, possibly due to low number of study participants. However, it is important to remember that although some of the cases did show clinically significant cataract some of the cataract extractions were performed primarily as a means to lower IOP and not to improve visual acuity.

Previous studies have shown that a certain proportion of eyes might develop non-functional or absence of previously implanted iStent inject microstents during follow-up. ${ }^{10}$ In the 
current study one such case was suspected (intraoperatively) in which case an additional microstent was implanted at the same occasion (three in total). Interestingly, a study by Gillmann et al on combined iStent inject implantation showed that $72 \%$ of devices were not situated within the Schlemm's canal. It is not unlikely that this might have contributed to the poorer outcome in the iStent inject subgroups. ${ }^{24}$

Furthermore, postoperative IOP elevation is a known complication following stent implantation. ${ }^{4,19}$ In this study, one case of sustained IOP elevation $(\leq 28 \mathrm{mmHg}$ ) occurred shortly after the procedure resulting in a subsequential KDB goniotomy six months later after which IOP remained within an acceptable range.

A strength as well as weakness of the present study was that one single experienced surgeon was responsible for all surgeries as well as oversaw the postoperative treatment for all patients. However, this might also have introduced a certain degree of sampling bias. Another strength is the direct comparison of two canal-based glaucoma procedures both as standalone procedures or combined with cataract extraction since such head-to-head comparisons are greatly lacking in currently published literature. Being a small and retrospective pilot study the current study posed several shortcomings. The lack of randomization was one of the main weaknesses of this study. Even if the preoperative indications for surgery were similar between the groups, allocating patients to either of the surgical techniques depended mainly on the surgeon's preferences. Furthermore, by including only mild-moderate severity types of glaucoma means that the results of this study might not necessarily apply to more severe types of glaucoma. Finally, the relatively small sample size precludes any far-reaching conclusions.

\section{Conclusion}

This study shows a clinically meaningful effect in reducing IOP and number of IOP-lowering medications in both the single and combined KDB goniotomy subgroups as well as the combined iStent inject subgroup. Although certain shortcomings exist in the present study that might work in favor of $\mathrm{KDB}$, data from this study do indicate that excisional goniotomy with Kahook Dual Blade might offer a favorable IOP reduction in comparison to iStent inject implantation. Both techniques demonstrate a convincingly favorable safety profile without any sight-threatening complications.

\section{Disclosure}

Dr. MA Economou has received lecture fees from Glaukos Corp. and New World Medical Inc. No other author has a financial or proprietary interest in any material or method mentioned.

\section{References}

1. Tham YC, Li X, Wong TY, et al. Global prevalence of glaucoma and projections of glaucoma burden through 2040: a systematic review and meta-analysis. Ophthalmology. 2014;121(11):2081-2090. doi:10.1016/ j.ophtha.2014.05.013

2. Pillunat LE, Erb C, Junemann AG, et al. Micro-invasive glaucoma surgery (MIGS): a review of surgical procedures using stents. Clin Ophthalmol. 2017;11:1583-1600. doi:10.2147/OPTH.S135316

3. Fea AM. Phacoemulsification versus phacoemulsification with micro-bypass stent implantation in primary open-angle glaucoma: randomized double-masked clinical trial. J Cataract Refract Surg. 2010;36(3):407-412. doi:10.1016/j.jcrs.2009.10.031

4. Voskanyan L, Garcia-Feijoo J, Belda JI, et al. Prospective, unmasked evaluation of the iStent(R) inject system for open-angle glaucoma: synergy trial. Adv Ther. 2014;31(2):189-201. doi:10.1007/s12325-0140095-y

5. Arriola-Villalobos P, Martinez-de-la-Casa JM, Diaz-Valle D, et al. Glaukos iStent inject(R) trabecular micro-bypass implantation associated with cataract surgery in patients with coexisting cataract and open-angle glaucoma or ocular hypertension: a long-term study. J Ophthalmol. 2016;2016. doi:10.1155/2016/1056573

6. Greenwood MD, Seibold LK, Radcliffe NM, et al. Goniotomy with a single-use dual blade: short-term results. J Cataract Refract Surg. 2017;43(9):1197-1201. doi:10.1016/j.jcrs.2017.06.046

7. Salinas L, Chaudhary A, Berdahl JP, et al. Goniotomy using the Kahook Dual Blade in severe and refractory glaucoma: six month outcomes. J Glaucoma. 2018;27(10):849-855. doi:10.1097/IJG.0000000000001019

8. Seibold LK, Soohoo JR, Ammar DA, et al. Preclinical investigation of ab interno trabeculectomy using a novel dual-blade device. $\mathrm{Am}$ J Ophthalmol. 2013;155(3):524-529.e2. doi:10.1016/j.ajo.2012.09.023

9. Ting JL, Damji KF, Stiles MC. Ab interno trabeculectomy: outcomes in exfoliation versus primary open-angle glaucoma. J Cataract Refract Surg. 2012;38(2):315-323. doi:10.1016/j.jcrs.2011.08.043

10. Arriola-Villalobos P, Martinez-de-la-Casa JM, Diaz-Valle D, et al. Mid-term evaluation of the new Glaukos iStent with phacoemulsification in coexistent open-angle glaucoma or ocular hypertension and cataract. Br J Ophthalmol. 2013;97(10):1250-1255. doi:10.1136/ bjophthalmol-2012-302394

11. Arnljots TS, Economou MA. Reversible cystoid macular edema following uneventful microinvasive Kahook Dual Blade goniotomy in a pseudophakic patient: a case report. J Glaucoma. 2018;27(7):e128-e130. doi:10.1097/IJG.0000000000000965

12. Sieck EG, Epstein RS, Kennedy JB, et al. Outcomes of Kahook Dual Blade goniotomy with and without phacoemulsification cataract surgery. Ophthalmology. 2018;1(1):75-81. doi:10.1016/j.ogla.2018.06.006

13. Kornmann HL, Fellman RL, Feuer WJ, et al. Early results of goniotomy with the Kahook Dual Blade, a novel device for the treatment of glaucoma. Clin Ophthalmol. 2019;13:2369-2376. doi:10.2147/OPTH. S224643

14. ElMallah MK, Seibold LK, Kahook MY, et al. 12-month retrospective comparison of Kahook Dual Blade excisional goniotomy with iStent trabecular bypass device implantation in glaucomatous eyes at the time of cataract surgery. Adv Ther. 2019;36(9):2515-2527. doi:10.1007/s12325-019-01025-1

15. Le C, Kazaryan S, Hubbell M, et al. Surgical outcomes of phacoemulsification followed by iStent implantation versus goniotomy with the Kahook Dual Blade in patients with mild primary open-angle glaucoma with a minimum of 12-month follow-up. J Glaucoma. 2019;28(5):411-414. doi:10.1097/ IJG.0000000000001143 
16. Dorairaj SK, Kahook MY, Williamson BK, et al. A multicenter retrospective comparison of goniotomy versus trabecular bypass device implantation in glaucoma patients undergoing cataract extraction. Clin Ophthalmol. 2018;12:791-797. doi:10.2147/OPTH.S158403

17. Davids AM, Pahlitzsch M, Boeker A, et al. iStent inject as a reasonable alternative procedure following failed trabeculectomy? Eur J Ophthalmol. 2018;28(6):735-740. doi:10.1177/1120672117747010

18. Klamann MK, Gonnermann J, Pahlitzsch M, et al. iStent inject in phakic open angle glaucoma. Graefes Arch Clin Exp Ophthalmol. 2015;253(6):941-947. doi:10.1007/s00417-015-3014-2

19. Fea AM, Belda JI, Rekas M, et al. Prospective unmasked randomized evaluation of the iStent inject ((R)) versus two ocular hypotensive agents in patients with primary open-angle glaucoma. Clin Ophthalmol. 2014;8:875-882. doi:10.2147/OPTH.S59932

20. Gonnermann J, Bertelmann E, Pahlitzsch M, et al. Contralateral eye comparison study in MICS \& MIGS: trabectome(R) vs. iStent inject(R). Graefes Arch Clin Exp Ophthalmol. 2017;255(2):359-365. doi:10.1007/s00417-016-3514-8

21. Samuelson TW, Sarkisian SR Jr, Lubeck DM, et al. Prospective, randomized, controlled pivotal trial of an ab interno implanted trabecular micro-bypass in primary open-angle glaucoma and cataract: two-year results. Ophthalmology. 2019;126(6):811-821. doi:10.1016/j.ophtha.2019.03.006
22. Katz LJ, Erb C, Carceller Guillamet A, et al. Long-term titrated IOP control with one, two, or three trabecular micro-bypass stents in open-angle glaucoma subjects on topical hypotensive medication: 42-month outcomes. Clin Ophthalmol. 2018;12:255-262. doi:10.2147/OPTH.S152268

23. Iwasaki K, Takamura Y, Orii Y, et al. Performances of glaucoma operations with Kahook Dual Blade or iStent combined with phacoemulsification in Japanese open angle glaucoma patients. Int J Ophthalmol. 2020;13(6):941-945. doi:10.18240/ijo.2020.06.13

24. Gillmann K, Bravetti GE, Mermoud A, et al. A prospective analysis of iStent inject microstent positioning: Schlemm canal dilatation and intraocular pressure correlations. J Glaucoma. 2019;28(7):613-621. doi:10.1097/IJG.0000000000001273
Clinical Ophthalmology

\section{Publish your work in this journal}

Clinical Ophthalmology is an international, peer-reviewed journal covering all subspecialties within ophthalmology. Key topics include: Optometry; Visual science; Pharmacology and drug therapy in eye diseases; Basic Sciences; Primary and Secondary eye care; Patient Safety and Quality of Care Improvements. This journal is indexed on PubMed

\section{Dovepress}

Central and CAS, and is the official journal of The Society of Clinical Ophthalmology (SCO). The manuscript management system is completely online and includes a very quick and fair peer-review system, which is all easy to use. Visit http://www.dovepress.com/ testimonials.php to read real quotes from published authors. 